\title{
Costs, outcome and cost-effectiveness of neurocritical care: a multi-center observational study
}

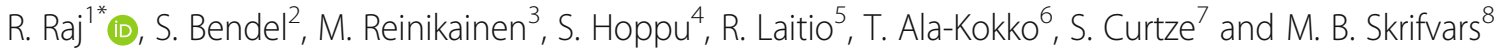

\begin{abstract}
Background: Neurocritical illness is a growing healthcare problem with profound socioeconomic effects. We assessed differences in healthcare costs and long-term outcome for different forms of neurocritical illnesses treated in the intensive care unit (ICU).

Methods: We used the prospective Finnish Intensive Care Consortium database to identify all adult patients treated for traumatic brain injury $(\mathrm{TBI})$, intracerebral hemorrhage $(\mathrm{ICH})$, subarachnoid hemorrhage $(\mathrm{SAH})$ and acute ischemic stroke (AIS) at university hospital ICUs in Finland during 2003-2013. Outcome variables were one-year mortality and permanent disability. Total healthcare costs included the index university hospital costs, rehabilitation hospital costs and social security costs up to one year. All costs were converted to euros based on the 2013 currency rate.

Results: In total 7044 patients were included (44\% with TBI, 13\% with ICH, 27\% with SAH, 16\% with AIS). In comparison to $\mathrm{TBI}, \mathrm{ICH}$ was associated with the highest risk of death and permanent disability (OR 2.6, 95\% Cl 2.1-3.2 and OR 1.7, 95\% $\mathrm{Cl} 1.4-2.1)$, followed by AIS (OR 1.9, 95\% Cl 1.5-2.3 and OR 1.5, 95\% Cl 1.3-1.8) and SAH (OR 1.8, 95\% Cl 1.5-2.1 and OR 0. $8,95 \% \mathrm{Cl} 0.6-0.9)$, after adjusting for severity of illness. SAH was associated with the highest mean total costs (€51,906) followed by ICH (€47,661), TBI (€43,916) and AIS (€39,222). Cost per independent survivor was lower for TBI $(€ 58,497)$ and SAH (€96,369) compared to AIS (€104,374) and ICH (€178,071).

Conclusion: Neurocritical illnesses are costly and resource-demanding diseases associated with poor outcomes. Intensive care of patients with TBI or SAH more commonly result in independent survivors and is associated with lower total treatments costs compared to $\mathrm{ICH}$ and AIS.
\end{abstract}

Keywords: Neurocritical care; neurointensive care; costs, Outcome; cost-effectiveness, Traumatic brain injury, Intracerebral hemorrhage, Acute ischemic stroke, Subarachnoid hemorrhage, Finland

\section{Background}

Neurocritical illnesses, including traumatic brain injury (TBI), intracerebral hemorrhage (ICH), subarachnoid hemorrhage (SAH) and acute ischemic stroke (AIS), are major killers and impose a growing socioeconomic burden around the world [1-4]. The most severe illnesses or injuries are treated at specialized intensive care units (ICU). Intensive care is, however, very resource-demanding, comprising for example almost $0.7 \%$ of the national gross domestic product in the USA [5]. Furthermore, the need

\footnotetext{
* Correspondence: rahul.raj@hus.fi

${ }^{1}$ Department of Neurosurgery, Helsinki University Hospital and University of Helsinki, Topeliuksenkatu 5, PB 266, 00029 HUS Helsinki, Finland

Full list of author information is available at the end of the article
}

costs of intensive care beds appears to continue to rise [5-8]. Due to limited healthcare budgets clinicians and hospital administrators are interested in measuring and evaluating intensive care cost-effectiveness. Patients with neurocritical illnesses constitute approximately $20-25 \%$ of severely ill patients requiring intensive care (unpublished data from the Finnish Intensive Care Consortium). Still, cost-analysis studies of neurocritical illnesses are lacking [9].

Finland offers a unique setting to comprehensively assess healthcare costs. Finland has a public tax-funded healthcare system, allowing every citizen to be equally treated at the right level of care at every step (including acute care and rehabilitation), independent of for example

(C) The Author(s). 2018 Open Access This article is distributed under the terms of the Creative Commons Attribution 4.0 International License (http://creativecommons.org/licenses/by/4.0/), which permits unrestricted use, distribution, and reproduction in any medium, provided you give appropriate credit to the original author(s) and the source, provide a link to the Creative Commons license, and indicate if changes were made. The Creative Commons Public Domain Dedication waiver (http://creativecommons.org/publicdomain/zero/1.0/) applies to the data made available in this article, unless otherwise stated. 
socioeconomic factors and insurance status. In regard to neurocritical disease, all specialized neurointensive care has been centralized to five university hospitals for several decades. Thus, all the most severe and resourcedemanding cases are treated at the university hospital level, enabling comprehensive cost-analysis studies. Furthermore, the Social Insurance Institution (Kela) in Finland covers all Finnish citizens regardless of personal insurance status.

The aim of this study is to describe differences in total one-year healthcare costs and one-year mortality and treatment cost-effectiveness following ICU admission after neurocritical disease (TBI, ICH, SAH and AIS). Especially, we aimed to study treatment cost-effectiveness of independent survivors.

\section{Methods}

\section{Study design and study population}

Finland has a publicly funded three-tier healthcare system, where five university hospitals provide the highest level of care, including specialized neurointensive care. We used the Finnish Intensive Care Consortium (FICC) database to include all adult patients (age $\geq 18$ years) treated for TBI, ICH, SAH or AIS in the five university hospitals in Finland from 2003 to 2013. The FICC database is a nationwide prospective data-collecting database including all ICU-treated patients from the majority of all ICUs in Finland [10].

We defined TBI, ICH, SAH and AIS according to the Acute Physiology and Chronic Health Evaluation III (APACHE III) diagnosis and International Classification of Diseases and Related Health Problems, 10th Revision (ICD-10) diagnosis. We confirmed the TBI and ICH diagnoses by reviewing the patients' computerized tomography (CT) head scans obtained on admission. ICH and SAH diagnoses do not include those due to trauma (these are coded as TBI). We excluded patients with missing baseline data (non-emergency admission, missing Glasgow Coma Scale (GCS) score, missing pre-admission functional status, missing cost data, see Fig. 1).

\section{Extracted variables}

We extracted the following variables from the FICC database: age, GCS score, pre-admission functional status, significant chronic comorbidity, mechanical ventilation, length of ICU stay, length of hospital stay, Therapeutic Intervention Scoring System 76 (TISS-76 [11]), APACHE II [12], Simplified Acute Physiological Score II (SAPS II [13]) and first-day Sequential Organ Failure Assessment (SOFA [14]) score. In FICC, the GCS score is defined as the worst measured GCS score during the first ICU day or the last reliable GCS was used for intubated and/or sedated patients according to the SAPS II definition [13]. Pre-admission functional status is a modified version of

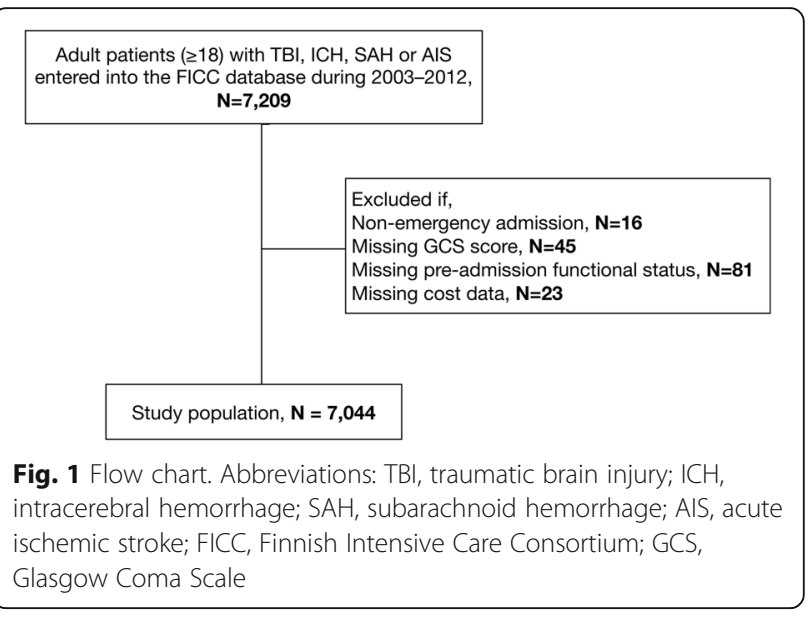

the World Health Organization/Eastern Cooperative Oncology (WHO/ECOG) classification used in the FICC [15]. Significant chronic comorbidity is defined according to the APACHE II and SAPS II definitions [12, 13]. The TISS-76 score includes 76 items measuring nursing staff workload and the intensity of given treatments. It was originally developed as a tool to quantify the workload and treatment intensity required by ICU-treated patients, but has since also been used as a dynamic measure of patient prognosis [16].

\section{Definition of outcome variables}

We used one-year mortality and a surrogate marker for permanent disability as outcome variables. We obtained data on date of death through the Finnish population register (available for all patients) and the archive of death certificates. We defined patients permanently disabled if they were granted a permanent disability allowance or disability pension by the Social Insurance Institution (Kela) by 30 September 2016. The criteria for Kela to grant permanent disability allowance or disability pension is being unable to independently carry out daily activities (e.g. self-hygiene, basic housekeeping, take care of things outside the home) or to be unable to return to work, for a minimum of one consecutive year. The Kela criteria for granting permanent disability allowances did not change during the study period [17]. We also report hospital mortality rates.

\section{Definition of cost variables}

The total healthcare cost variable consists of three parameters: university hospital costs, rehabilitation hospital costs and social security costs up to one year after the index admission [17]. We obtained university hospital costs directly from the hospitals' billing departments. University hospital costs includes expenses from the whole treatment period, including e.g. personnel costs, surgery, ICU stay, laboratory costs, radiological costs, and costs of ward stay. 
We calculated rehabilitation hospital costs by multiplying the length of rehabilitation stay by the average price of one ward day in the designated level-of-care unit, according to a report from the Finnish National Institute for Health and Welfare [18]. We obtained social security costs directly from Kela. Social security costs include for example disability allowances, sickness allowances, private physician and physiotherapist expenses, prescription drug expenses and medical travel costs. In Finland, Kela is a Finnish government agency funded by tax money, insurance payments, and municipalities that provide all social security payments in Finland. Thus, social security payments are not dependent upon, for example, personal insurance. We adjusted all costs according to the Consumer Price Index (CPI) in Finland to euros based on the currency rate in 2013:

$$
\text { CPI adjusted cost }=\text { Cost } * \frac{\text { CPI in } 2013}{\text { Admission year CPI }}
$$

\section{Statistics}

We used SPSS Statistics 24.0 for mac OS (IBM Corp, Armonk, NY, USA) and Stata Statistical Software for mac OS (StataCorp LP, College Station, TX, USA) for the statistical analyses. We compared categorical variables using the chi-squared $\left(x^{2}\right)$ test with the Bonferroni correction. We tested continuous data for skewness and as all variables were highly skewed they are reported as medians with interquartile ranges (IQR), unless other specified (such as cost data). We compared non-parametric continuous variables between groups using a non-parametric test. We present cost data as means with 95\% confidence intervals (CI) and compared means between groups using the $t$ test.

To compare costs adjusted for severity of illness between the different diagnostic groups we created a severity of illness model using multivariate logistic regression analysis, with one-year mortality and permanent disability (for one-year survivors) as the dependent variable, separately for the diagnostic groups to weight the predictors according to the diagnostic group. The severity of illness model included variables that were significantly $(p \leq 0.05)$ associated with one-year mortality in univariate analysis. These were the same for all diagnoses: age, GCS score, chronic comorbidity, pre-admission functional status and SAPS II score (excluding age, GCS score, and chronic comorbidity). SAPS II was chosen over APACHE II in accordance with findings from our previous studies $[19,20]$. The predictive ability of the severity of illness model was assessed by the area under the receiver operating curve (AUC).

We then used multivariate linear regression analysis (regress in Stata), adjusting for variables in the severity of illness model to assess differences in costs between the diagnostic groups. We also used the same model in a multilevel mixed-effect logistic regression model (melogit in Stata) to assess differences in risk of one-year mortality and permanent disability between the diagnostic groups. Diagnosis (TBI, ICH, SAH, AIS) was modeled as a random effect and considered as the random part of the intercept, as the effect of the included variables may be different in respective diagnostic groups.

To evaluate cost-effectiveness, we calculated the effective cost per survivor (ECPS) and effective cost per independent survivor (ECPIS), which is defined as the cost for all patients divided by the number of one-year survivors or independent one-year survivors [21]. As a sensitivity analysis we divided patients into four risk bands according to probability $(0-25 \%, 26-50 \%, 51-75 \%, 76-100 \%)$ of one-year mortality within the diagnostic group. We conducted the study according to the Strengthening the reporting of observational studies in epidemiology (STROBE) guidelines (Additional file 1).

\section{Results}

The final study population included 7044 patients (Fig. 1). Of these, 3097 had TBI (43\%); 949 had ICH (14\%); 1875 had SAH (27\%) and 1123 had AIS (16\%). Patient baseline characteristics are shown in Table 1. Patients with AIS or ICH were older those with than TBI or SAH, respectively. The GCS score was lower among patients with TBI or ICH compared to SAH or AIS. Patients were predominantly female only in the group of patients with SAH. Patients with $\mathrm{SAH}$ had the best pre-admission functional status. There were no major differences in significant comorbidities between the diagnostic groups. Patients with SAH had the longest ICU and hospital length of stay. Mean daily treatment intensity measured using the TISS-76 was similar in patients with TBI, ICH or SAH but lower among patients with AIS. Total treatment intensity was notably higher in patients with SAH compared to the other groups. Patients with ICH had the highest SAPS II and APACHE II scores followed by patients with TBI, SAH or AIS.

\section{Severity of illness models}

The AUC for the severity of illness model, for one-year mortality prediction, including all patients, was 0.86 (95\% CI $0.85-0.87$ ), indicating good discrimination. The AUC was 0.85 (95\% CI $0.84-0.87$ ) for patients with TBI, for ICH the AUC was 0.85 (95\% CI 0.82-0.87), for SAH the AUC was 0.89 (95\% CI $0.87-0.90)$ and for AIS the AUC was 0.88 (95\% CI $0.85-0.90)$. The AUC for the severity of illness model, for permanent disability prediction (for one-year survivors), including all patients, was 0.71 (95\% CI $0.70-0.73$ ), indicating satisfactory discrimination. The AUC was 0.73 (95\% CI 0.70-0.75) for patients with TBI, 
Table 1 Patient baseline characteristics

\begin{tabular}{|c|c|c|c|c|c|}
\hline Variables & TBI $(N=3097)$ & $\mathrm{ICH}(\mathrm{N}=949)$ & $\mathrm{SAH}(N=1875)$ & $\operatorname{AIS}(N=1123)$ & $P$ value \\
\hline Age (median, IQR) & $56(41-67)$ & $61(52-69)$ & $56(47-65)$ & $68(59-76)$ & $<0.001$ \\
\hline $18-40$ years & $750(24 \%)$ & $71(7 \%)$ & $193(10 \%)$ & $42(4 \%)$ & $<0.001$ \\
\hline $41-64$ years & 1455 (47\%) & $509(54 \%)$ & $1201(64 \%)$ & $420(37 \%)$ & \\
\hline$\geq 65$ years & $892(29 \%)$ & 369 (39\%) & $481(26 \%)$ & $661(59 \%)$ & \\
\hline GCS score, median (IQR) & $9(5-14)$ & $8(4-13)$ & $12(5-15)$ & $12(8-15)$ & $<0.001$ \\
\hline $3-8$ & $1465(47 \%)$ & 497 (52\%) & 704 (38\%) & $313(28 \%)$ & $<0.001$ \\
\hline $9-12$ & $598(20 \%)$ & $172(18 \%)$ & $262(14 \%)$ & $253(22 \%)$ & \\
\hline $13-15$ & 1034 (33\%) & $280(30 \%)$ & 909 (48\%) & $557(50 \%)$ & \\
\hline Female & $711(23 \%)$ & $332(35 \%)$ & 1065 (57\%) & $443(39 \%)$ & $<0.001$ \\
\hline Pre-admission functional status ${ }^{a}$ & & & & & $<0.001$ \\
\hline Fit for work or equal & $1896(61 \%)$ & $613(65 \%)$ & $1473(79 \%)$ & $717(64 \%)$ & \\
\hline Unfit for work, but independent in self-care & $967(31 \%)$ & $252(27 \%)$ & $332(18 \%)$ & $275(24 \%)$ & \\
\hline Partially dependent in self-care & $180(6 \%)$ & $61(6 \%)$ & $47(2 \%)$ & $110(10 \%)$ & \\
\hline Totally dependent in self-care & $54(2 \%)$ & $23(2 \%)$ & $23(1 \%)$ & $21(2 \%)$ & \\
\hline Significant chronic comorbidity ${ }^{\mathrm{b}}$ & $267(9 \%)$ & $108(11 \%)$ & $154(8 \%)$ & $138(12 \%)$ & $<0.001$ \\
\hline Mechanical ventilation & $2070(67 \%)$ & $640(67 \%)$ & $1241(66 \%)$ & $427(38 \%)$ & $<0.001$ \\
\hline LOS ICU, days (median, IQR) & $2(1-4)$ & $2(1-3)$ & $3(1-6)$ & $1(1-2)$ & $<0.001$ \\
\hline LOS hospital, days (median, IQR) & $6(3-11)$ & $6(3-12)$ & $10(6-16)$ & $6(4-11)$ & $<0.001$ \\
\hline TISS-76 daily average ${ }^{c}$ (median, IQR) & $27(21-33)$ & $26(20-31)$ & $28(24-34)$ & $19(14-27)$ & $<0.001$ \\
\hline TISS-76 total ${ }^{C}$ (median, IQR) & $69(42-154)$ & $63(39-126)$ & $102(63-230)$ & $44(27-74)$ & $<0.001$ \\
\hline APACHE II score (median, IQR) & $19(13-25)$ & $20(13-26)$ & $17(11-24)$ & $14(9-22)$ & $<0.001$ \\
\hline SAPS II score (median, IQR) & $35(24-50)$ & $40(25-55)$ & $29(20-48)$ & $29(23-45)$ & $<0.001$ \\
\hline SOFA score ${ }^{d}$ (median, IQR) & $6(3-8)$ & $6(3-9)$ & $6(3-9)$ & $3(1-7)$ & $<0.001$ \\
\hline
\end{tabular}

Abbreviations: AIS, acute ischemic stroke; APACHE, Acute Physiology and Chronic Health Evaluation; GCS; Glasgow Coma Scale; ICH, intracerebral hemorrhage; LOS, length of stay; ICU, Intensive Care Unit; SAH, subarachnoid hemorrhage; SAPS, Simplified Acute Physiology Score; SOFA, Sequential Organ Failure Assessment; TBI, traumatic brain injury; TISS-76, Therapeutic Intervention Scoring System 76

${ }^{\mathrm{a}}$ A modified World Health Organization/Eastern Cooperative Oncology Group classification system implemented by the Finnish Intensive Care Consortium

${ }^{b}$ Any chronic comorbidity according to APACHE II or to SAPS II

${ }^{c}$ Missing for 6 patients

${ }^{\mathrm{d}}$ Missing for 9 patients

for $\mathrm{ICH}$ the AUC was 0.67 (95\% CI 0.62-0.71), for SAH the AUC was $0.72(95 \%$ CI $0.69-0.75)$ and for AIS the AUC was 0.68 (95\% CI 0.64-0.72).

The overall probability of one-year mortality, reflecting severity of illness, decreased during the study period (Fig. 2). This decrease was most profound in patients with AIS or ICH (Additional file 2). With time the relative proportion of patients in the two lowest risk bands $(0-25 \%, 26-50 \%$ vs. $51-75 \%, 76-100 \%)$ became greater in all diagnostic groups but especially in the $\mathrm{ICH}$ and AIS groups (Additional file 3).

\section{Patient outcome}

Patient outcome is shown in Table 2. Hospital mortality was highest among patients with ICH (28\%), followed by SAH (18\%), AIS (16\%) and TBI (13\%). One-year mortality was highest among patients with $\mathrm{ICH}(44 \%)$ followed by AIS (29\%), SAH $(27 \%)$ and TBI (25\%). In a multilevel mixed-effect logistic regression model, using TBI as the reference group, ICH was associated with the highest risk of one-year mortality (OR 2.61, 95\% CI 2.15-3.16) followed by AIS (OR 1.90, 95\% CI 1.55-2.33) and SAH (OR 1.79, 95\% CI 1.51-2.11).

Permanent disability in one-year survivors was highest among patients with ICH (51\%), followed by AIS (47\%), TBI (37\%) and SAH (26\%). In the multilevel mixed-effect logistic regression model, using TBI as the reference group, ICH was associated with the highest risk of permanent disability (OR 1.75, 95\% CI 1.42-2.14) followed by AIS (OR 1.53, 95\% CI 1.27-1.85). In contrast, SAH was associated with a lower risk of permanent disability compared to TBI (OR for SAH in comparison to TBI was 0.75, 95\% CI 0.64-0.88).

\section{Costs and cost-effectiveness}

Overall mean cost per patient was €45,799 (95\% CI $€ 44,597-€ 47,001)$ out of which $45 \%$ constituted university hospital costs (mean €20,493, 95\% CI 19,945-21,040), 39\% 


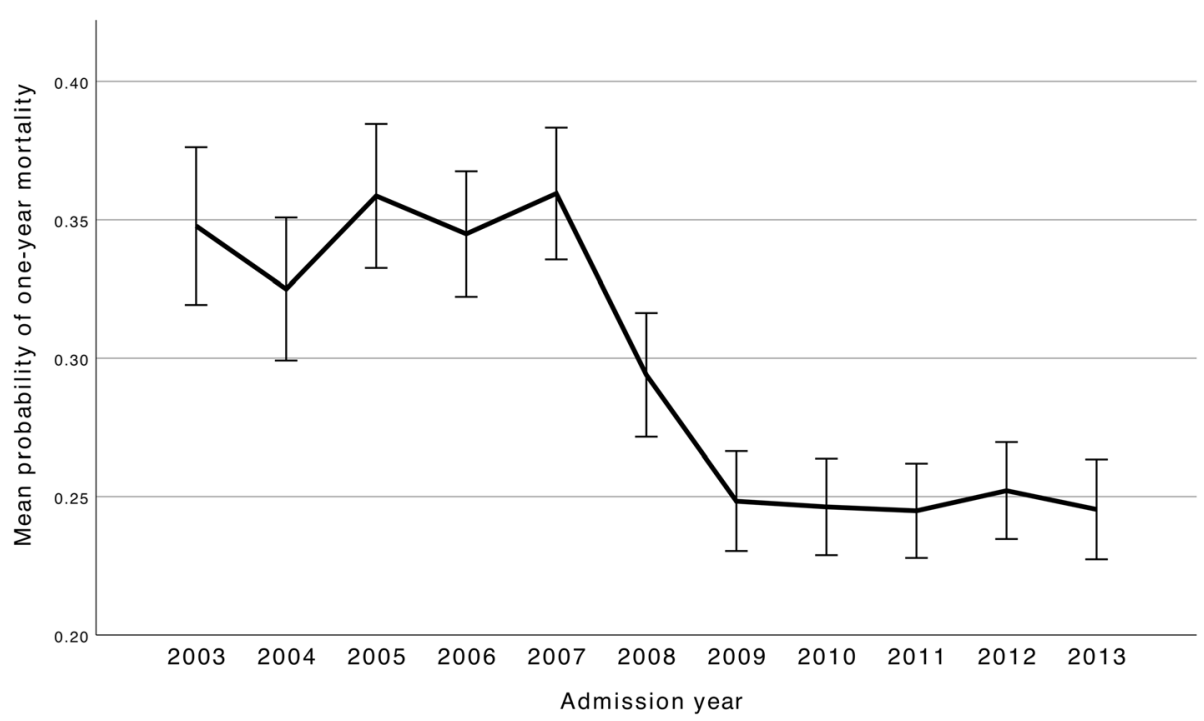

Fig. 2 Changes in mean probability of one-year mortality (with 95\% confidence intervals), reflecting patient severity of illness. Probabilities are calculated by logistic regression analysis, adjusting for age, Glasgow Coma Scale score, significant comorbidity, pre-admission functional status and the modified Simplified Acute Physiology Score II. The $y$-axis scale extends from 0 to 0.4 , where 0 indicates that the probability is $0 \%$ and 0.4 that the probability is $40 \%$. Severity of illness decreased markedly from 2007 to 2009 , where after it remained largely the same

rehabilitation hospital costs (mean €18,064, 95\% CI $€ 17,245-18,883$ ) and $16 \%$ social security costs (mean $€ 7242,95 \%$ CI $€ 6992-€ 7492)$.

Differences in costs between the diagnostic groups are shown in Table 2. University hospital costs were notably higher for patients with SAH compared to the other diagnostic groups ( $€ 25,717$ vs. $€ 15,819-€ 19,568)$. Rehabilitation costs were similar between the groups, being slightly higher for patients with TBI or ICH compared to SAH or AIS ( $€ 18,435$ and $€ 21,361$ compared to $€ 16,673$ and $€ 16,579)$. Social security costs were highest for patients with SAH and lowest for patients with TBI (€9516 vs. €5913).
Change in the sum of costs for all patients is shown in Fig. 3. In 2003, the sum of costs for all patients was $€ 18.6$ million. In 2013, this sum had risen to $€ 32.7$ million or on average by $7 \%$ per year. Still, the mean cost per patient decreased on average by $2 \%$ from 2003 to 2013 (Fig. 3). Changes in the sum of costs for the different diagnostic groups is shown in Additional file 4. The sum of costs increased notably in all groups with the most marked relative increase in patients with AIS. Additional file 5 shows changes in mean cost per patient during the study period. On average, the mean cost per patient decreased in patients with TBI or ICH, remained

Table 2 Mean costs and unadjusted outcomes by diagnosis

\begin{tabular}{|c|c|c|c|c|c|}
\hline Outcome & TBI $(N=3097)$ & $\mathrm{ICH}(N=949)$ & $\mathrm{SAH}(N=1875)$ & AIS $(N=1123)$ & $P$ value \\
\hline Hospital mortality & $12.5 \%(11.3-13.6)$ & $27.6 \%(24.8-30.5)$ & $18.3 \%(16.5-20.0)$ & $16.4 \%(14.2-18.6)$ & $<0.001$ \\
\hline One-year mortality & $24.7 \%(23.2-26.2)$ & $43.8 \%(40.7-47.0)$ & $27.1 \%(25.1-29.1)$ & $28.7 \%(26.0-31.3)$ & $<0.001$ \\
\hline Permanent disability ${ }^{a}$ & $36.7 \%(34.8-38.7)$ & $51.0 \%(46.8-55.3)$ & $26.0 \%(23.7-38.4)$ & $46.9 \%(43.5-50.4)$ & $<0.001$ \\
\hline \multicolumn{6}{|l|}{ Mean costs } \\
\hline University hospital costs & $€ 19,568(18,707-20,429)$ & $€ 18,721(17,125-20,316)$ & $€ 25,717(24,722-26,713)$ & $€ 15,819(14,665-16,972)$ & $<0.001$ \\
\hline Percentage of total costs & $45 \%$ & $39 \%$ & $50 \%$ & $40 \%$ & \\
\hline Rehabilitation hospital & $€ 18,435(17,164-19,706)$ & $€ 21,361(19,055-23,667)$ & $€ 16,673(15,112-18,234)$ & $€ 16,579(14,720-18,437)$ & $<0.001$ \\
\hline Percentage of total costs & $42 \%$ & $45 \%$ & $32 \%$ & $42 \%$ & \\
\hline Social security costs & $€ 5913$ (5608-6217) & $€ 7579$ (6791-8367) & $€ 9516(8975-10,057)$ & $€ 6824$ (6158-7491) & $<0.001$ \\
\hline Percentage of total costs & $13 \%$ & $16 \%$ & $18 \%$ & $17 \%$ & \\
\hline Total costs & $€ 43,916(42,096-45,735)$ & $€ 47,661(44,198-51,123)$ & $€ 51,906(49,544-54,268)$ & $€ 39,222(36,541-41,903)$ & $<0.001$ \\
\hline
\end{tabular}

Outcome data presented as percentages with $95 \%$ confidence intervals (CI). Cost data presented as means with $95 \% \mathrm{Cls}$

Abbreviations: AIS, acute ischemic stroke; ICH, intracerebral hemorrhage; SAH, subarachnoid hemorrhage; $\mathrm{TBI}$, traumatic brain injury

Costs shown in euros $(€)$ at the 2013 rate

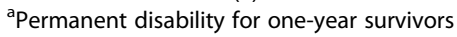



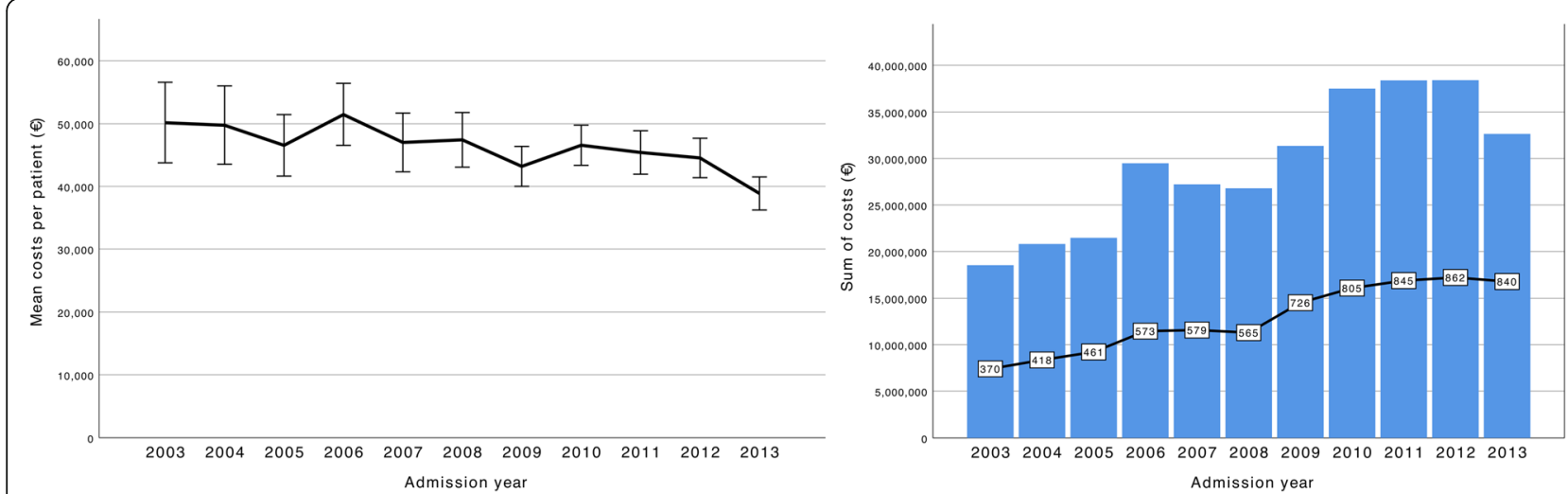

Fig. 3 Left panel, changes in mean costs per patient during the study period (with 95\% confidence intervals (CI)]). A trend towards lower mean costs per patient is noted. Mean cost per patient was €50,162 (95\% Cl €43,783-€56,541) in 2003 and $€ 38,872(95 \% \mathrm{Cl} € 36,236-€ 41,508)$ in 2013. Right panel, changes in the sum of costs (blue bars) and absolute number of patients per year (connected boxes). The sum of costs increased by 76\% from 2003 to 2013 (€18.6 million in 2003 and €32.8 million in 2013). The total number of patients increased by $227 \%$ from 370 patients in 2003 to 840 patients in 2013

unchanged in patients with AIS and increased in patients with SAH.

In the linear regression model, adjusting for the severity of illness model using TBI as the reference population, SAH was associated with the highest costs $(€ 7761,95 \%$ CI $€ 4813-€ 10,709, p<0.001)$, followed by ICH ( $€ 4044,95 \%$ CI $€ 305-€ 7782, p=0.034)$. On the contrary, AIS was associated with a lower total cost compared to TBI $(-4861$ euros, $95 \% \mathrm{CI}-8365$ to -1357 euros, $p=0.007$ ).

In regard to cost-effectiveness, AIS had the lowest ECPS, followed by TBI, SAH and ICH. However, the ECPIS for TBI and SAH was lower than for AIS and $\mathrm{ICH}$ (Fig. 4). In the sensitivity analysis, TBI and SAH had the lowest ECPIS in all risk bands (Additional file 6). In the most severely ill patients in risk band 4, TBI and AIS had the lowest ECPS, while TBI and SAH had the lowest ECPIS. ICH was associated with the lowest cost-effectiveness in all risk bands.

\section{Discussion}

\section{Principal findings}

In this comprehensive cost-analysis study, we found that the burden of ICU-treated TBI, ICH, SAH and AIS has markedly increased during the past decade in Finland. This is mainly due to an increased number of patients requiring neurocritical care being admitted to the ICU, as mean cost per patient slightly decreased during the study period. The decrease in mean cost per patient probably reflects the fact that severity of illness was less in all diagnostic groups, indicating that more patients with less severe neurocritical illness are treated in the ICU nowadays. We also identified major differences in treatment costs and cost-effectiveness in patients with TBI, SAH, ICH or AIS. Intensive care of patients with TBI or SAH resulted more commonly in patients becoming independent survivors and was associated with lower treatment costs compared to ICH and AIS. That

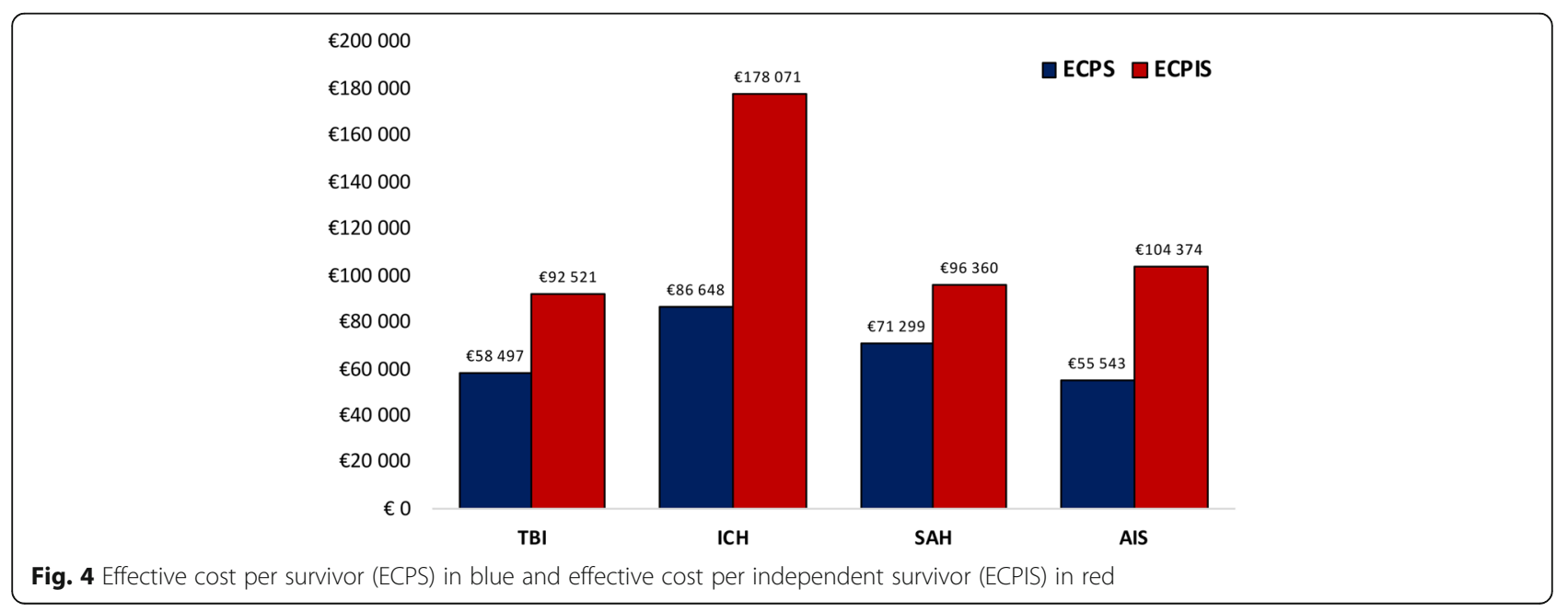


said, it should be noted that a direct comparison between patients with TBI, ICH, SAH or AIS is challenging due to the varied spectrum of patient demographics, natural history, ICU admission thresholds, treatment options and strategies and baseline prognosis.

\section{Comparison with other studies}

There are no studies that have comprehensively included several neurocritical illnesses. Regarding $\mathrm{ICH}$ and $\mathrm{SAH}$, a recent study from Ontario, showed that total direct and indirect hospital costs for patients with spontaneous ICH was $\$ 53,491$ (expressed in Canadian dollars at the 2017 rate, converts to $€ 35,303$ at the 2013 rate) and $\$ 92,794$ (expressed in Canadian dollars at the 2017 rate, converts to $€ 61,243$ at the 2013 rate) for patients with SAH [22]. The mean cost per SAH hospital survivor was $\$ 136,097$ (expressed in Canadian dollars at the 2017 rate, converts to $€ 89,797$ at the 2013 rate) and the mean cost per ICH hospital survivor was $\$ 94,856$ (expressed in Canadian dollars at the 2017 rate, converts to $€ 62,585$ at the 2013 rate). We found similar results, as the mean cost per one-year ICH was $€ 84,859$ (expressed in euros at the 2013 rate) and 71,195 (expressed in euros at the 2013 rate), per one-year SAH survivor.

Regarding SAH, a study from the UK in 2001, showed that the cost per life saved was $£ 40,816$ (expressed in pounds sterling at the 2001 rate, converts to $€ 82,546$ at the 2013 rate) and the cost to avoid one bad outcome was £84,366 (expressed in pounds sterling at the 2001 rate, converts to $€ 170,561$ at the 2013 rate) [23]. Quite similarly, the ECPS in our study was $€ 71,195$ (expressed in euros at the 2013 rate) but the ECPIS was markedly lower, being $€ 96,265$ (expressed at the 2013 rate). Another study from the UK reported that the estimated cost for treating one patient with SAH was $£ 23,294$ (expressed in pounds sterling at the 2005 rate, converts to $€ 38,701$ at the 2013 rate). This is somewhat lower than in our study, where the mean cost per patient with SAH was $€ 51,906$ (expressed in euros at the 2013 rate). Yet, the UK study did not include, for example, medication costs. In our study, the social security reimbursements were on average $€ 9516$ per patient with $\mathrm{SAH}$, making the results between our study and the UK study very similar.

SAH was associated with the highest university hospital costs whereas AIS was associated with the lowest university hospital costs. In contrast to the other diagnostic group, most patients with SAH undergo extensive and costly treatments, including aneurysm clipping or endovascular treatment [24]. The low university hospital costs in the AIS group may plausibly be explained by less severe illness (measured by the APACHE II and
SAPS II), fewer patients being mechanically ventilated, shorter lengths of stay and lower treatment intensity (measured by the TISS-76). SAH, on the other hand, was associated with the highest treatment intensity and longest length of stay, leading to higher university hospital costs. This and our previous study provide good data on the cost-effectiveness of intensive care in TBI [17]. Other TBI studies have identified total one-year direct and indirect costs between $€ 16,579$ and $€ 35,560$ (converted to euros at the 2013 rate) [25-27] but data on cost-effectiveness are lacking.

An inbuilt study bias is that ICU admission criteria vary among the diagnostic groups included. For example, patients with SAH are preferably directly admitted to the ICU in some centers, independent of their level of consciousness, while patients with TBI, ICH or AIS can be treated outside of the ICU, given an adequate level of consciousness and appropriate radiological findings. Further, patient age is probably key in ICU selection criteria, as elderly patients with stroke are often excluded from aggressive ICU treatment. This may distort our results towards improved cost-effectiveness, as some patients are admitted due to an estimated favorable outcome. It should also be mentioned that some of the patients included might be admitted to ICU due to potential organ donation. The American Heart Association/American Stroke Association recommends full treatment for at least 2 days before implementing any treatment limitations [28]. Organ donors often receive aggressive ICU treatment to uphold adequate organ vitality, which thus, is very resource-demanding as well. However, assessing the potential ICU treatment cost-effectiveness benefits of organ donation is out of the scope of this study.

We noted a marked decrease in unadjusted one-year mortality between 2007 and 2009. During the same years a substantial increase in patients admitted with $\mathrm{ICH}$ or AIS were noted due to structural changes in some centers leading to more less severely ill patients being admitted, which most likely explains the noted shift in mortality. It should also be highlighted that the vast majority of patients with ICH or AIS are treated outside of the ICU setting (for example in stroke units). In our study setting, only patients with the most severe cases of ICH and AIS are treated in the ICU, as all hospitals included have dedicated stroke units. Thus, our study obviously undervalues the total burden of AIS and ICH but represent the ICU-treated portion well. Furthermore, during the study period thrombectomy had not been established in the treatment of large vessel occlusion AIS [29-33]. The patients with and large vessel occlusion AIS probably constitute a notable proportion of patients with AIS being treated in the ICU prior to the establishment of thrombectomy. Thus, considering the effectiveness of thrombectomy for large vessel occlusion, 
the long-term cost-effectiveness of AIS has most likely improved and the number of patients with AIS requiring ICU care at all have may have decreased [34, 35]. This limits the interpretation and the generalization of the AIS results to the modern thrombectomy era. Cost-effectiveness correlated strongly with the severity of illness and the ECPIS was highest among patients with ICH or AIS in all risk bands, with the exception of risk band 4, where AIS was associated with the lowest ECPIS. Although we cannot confirm this, it is possible that these patients represent a group with relatively small infarcts but severe extracranial organ dysfunction (e.g. respiratory dysfunction after basilar artery occlusion), requiring intensive care, from which recovery is favorable.

\section{Future directions}

In our study we noted a steady increase in the number of neurocritical patients treated in the ICU. This probably reflects the parallel increase in ICU beds and the rapidly aging population in Finland, increasing the need for intensive care [8]. The total number of ICU beds in Finland is approximately 6 per 100,000 persons, which is among the lowest in Europe and one fifth of that in the USA [36, 37]. Thus, considering the rapidly aging population a continued increase in the number of patients requiring neurocritical care and an increased demand for ICU beds are to be expected. Accordingly, efforts should be put towards preventing these events, as outcomes are poor and treatment is resource-demanding and costly. Effectively treating risk factors for stroke $(\mathrm{SAH}, \mathrm{ICH}$, AIS) and TBI has the potential to reduce the burden of these neurocritical illnesses. For example, the decreased incidence of smoking in Finland has been linked to a decreased incidence in SAH [38]. Similar prevention should be targeted in TBI, which could be achieved by avoiding falls among the elderly, and reducing alcoholrelated falls and traffic-related accidents.

\section{Strengths and limitations}

This study was conducted in a government tax-funded healthcare system, where all citizens have the right to equal care, avoiding selection bias due to differences in access to treatment. Further, specialized neurointensive care has for decades been centralized to five university hospitals and, thus, the current study represents as good as the whole Finnish adult population. Moreover, data on rehabilitation hospital length of stay is electronically recorded on a national level by the Finnish National Institute for Health and Welfare, making this data extremely comprehensive. Another unique feature of this study is that in Finland, social security costs are essentially paid by one institution, Kela, which covers all Finnish citizens regardless of insurance status. Thus, in combination with the high-quality FICC database with the small number of missing patients, our study comprehensively catches the burden of neurocritical illnesses treated in the ICU in a developed country setting [10].

Regarding patients with TBI or SAH, we were able to include four of five ICUs providing specialized intensive care for these patients, making the TBI and SAH largely representative of the whole Finnish population, although less so than for ICH and AIS. Yet, as already mentioned, not all patients with AIS or ICH are treated in the ICU. After the introduction of thrombectomy the number of patients with AIS requiring ICU care has probably decreased as well. The noted increase in AIS admissions in this study is due to a change in local policy in one of the hospitals leading to more patients with AIS with less severe illness being treated in the ICU, probably explaining the decrease in unadjusted mortality in the middle of the study period. Thus, clearly our results on patients with AIS or ICH cannot be generalized to patients being treated outside the ICU.

We used a surrogate marker of permanent disability, i.e. if the patient was granted a permanent disability allowance or disability pension by Kela. In comparison to other commonly used outcome scales (e.g. Glasgow Outcome Scale, modified Rankin Scale), our definition may include some degree in inaccuracy but considering that all Finnish citizens are covered by Kela we believe that our definition adequately reflects neurological outcome. Further, the FICC database does not contain uniformly standardized data on limitation of care from the beginning of the study period. Thus, some included patients are probably ICU-treated due to upcoming potential organ donation.

We did only study adult patients and, thus, our results cannot directly be generalized to the pediatric population. Further, we only included patients with TBI, ICH, SAH or AIS. Thus, other neurocritical illnesses, such as cerebral venous thrombosis, status epilepticus, bacterial meningitis, viral encephalitis and neuromuscular disorders were not included. Last, the study was conducted in a single Nordic country. Thus, our results are best applicable to settings with similar healthcare systems.

\section{Conclusion}

Neurocritical illnesses are costly and resource-demanding diseases associated with poor outcomes. The total number of patients requiring neurocritical care who are admitted to the ICU has increased leading to an increase in total costs, although mean cost per patient has remained largely unchanged. These changes were paralleled with a decrease in patients' severity of illness. Intensive care of patients with TBI or SAH more commonly resulted in them becoming independent survivors and was associated with lower costs compared to the care of patients with ICH or AIS. 


\section{Additional files}

Additional file 1: The STROBE guidelines. (DOC $84 \mathrm{~kb}$ )

Additional file 2: Changes in probability of one-year mortality (with 95\% confidence intervals), reflecting severity of illness, for the diagnostic groups. The $y$-axis scale extends from 0 to 0.8 , where 0 indicates that the probability is $0 \%$ and 0.8 that the probability is $80 \%$. Probabilities were calculated by logistic regression analysis adjusting for age, GCS score, chronic comorbidity, pre-admission functional status and SAPS 2, separately for the diagnostic groups. A trend towards lower severity of illness was noted in all diagnostic groups. A small reduction in severity of illness was noted for patients with traumatic brain injury and subarachnoid hemorrhage. Between 2007 and 2009, severity of illness dropped notably in patients with acute ischemic stroke and intracerebral hemorrhage. (TIF $323 \mathrm{~kb}$ )

Additional file 3: Temporal change in risk bands within the diagnostic groups. Patients were divided into 4 equally sized risk bands within their own diagnostic group according to risk of one-year mortality (severity of illness). The relative proportion of patients in risk bands 1 and 2 increased with time in all diagnostic groups. The increase was most notable among patients with $\mathrm{ICH}$ and AIS, indicating that more patients with less severe illness were admitted towards the end of the study period. (TIF $544 \mathrm{~kb}$ )

Additional file 4: Changes in the sum of costs and absolute number of patients during the study period by diagnostic group. The bars (blue) represent the sum of costs for all patients in the particular diagnostic group treated during that particular year. The connected boxes indicate the absolute number of patients in the particular diagnostic group treated during that particular year. The absolute number of patients treated in the intensive care unit increased in all diagnostic groups. Accordingly, the sum of costs increased. (TIF $3003 \mathrm{~kb}$ )

Additional file 5: Changes in mean cost per patient (with 95\% confidence intervals) during the study period for the diagnostic groups. A trend towards lower mean cost per patient was noted in the traumatic brain injury, intracerebral hemorrhage and acute ischemic stroke groups, while mean cost per patient remained largely the same for patients in the subarachnoid hemorrhage group. (TIF $1051 \mathrm{~kb}$ )

Additional file 6: Effective cost per survivor (ECPS) and effective cost per independent survivor (ECPIS) according to diagnostic group and risk band. Risk band 1 (upper left) represents patients with one-year mortality risk of $0-25 \%$, risk band 2 (upper right) represents patients with one-year mortality risk of $26-50 \%$, risk band 3 (lower left) represents patients with one-year mortality risk of $51-75 \%$, and risk band 4 represent patients with one-year mortality risk of $76-100 \%$. The risk bands were created separately for all diagnostic groups by adjusting for age, GCS score, preadmission functional status, significant chronic comorbidity and the modified SAPS II score. (TIF $931 \mathrm{~kb}$ )

\section{Abbreviations}

AIS: Acute ischemic stroke; APACHE II: Acute Physiology and Chronic Health Evaluation II; AUC: Area under the receiver operating characteristic curve; CPI: Consumer Price Index; CT: Computed tomography; ECOG: Eastern Cooperative Oncology; ECPIS: Effective cost per independent survivor; ECPS: Effective cost per survivor; FICC: Finnish Intensive Care Consortium; GCS: Glasgow Coma Scale; ICH: Intracerebral hemorrhage; SAH: Subarachnoid hemorrhage; SAPS II: Simplified Acute Physiology Score II; SOFA: Sequential Organ Failure Assessment; STROBE: Strengthening the reporting of observational studies in epidemiology; TBI: Traumatic brain injury; TISS-76: Therapeutic Intervention Scoring System-76; WHO: World Health Organization

\section{Funding}

This study was funded with grants from Helsinki University (3-year project grant: H3702 73702705) and Helsinki University Hospital (state funding: VTRTYH2014221). RR has received personal research grants from Svenska Kulturfonden, Medicinska Understödsföreningen Liv and Hälsa, Finska Läkaresällskapet, Maud Kuistilan Säätiö, Eemil Aaltosen Säätiö, Ella and Georg Ehrnroothin Säätiö, Suomalais-Norjalainen Lääketieteen Säätiö, Suomen Lääketieteen Säätiö, and Maire Taposen Säätiö. The funders had no role in the study design, data collection and analysis, decision to publish or preparation of the manuscript.

\section{Availability of data and materials}

There are legal restrictions that prohibit us from making the data publicly available. The included data include a combination from several databases (The Finnish Intensive Care Consortium, Kela, the Finnish National Institute for Health and Welfare database, Statistics of Finland and the five university hospitals in Finland). Data can be requested directly from the sources, given appropriate research approval.

\section{Authors' contributions}

RR and MS had the main responsibility for study design and coordinating the study. RR, MS, MR and SB had the main responsibility in writing the manuscript. SH, RL, SC and TAK contributed to acquisition of data, data analysis, interpretation of data, and revision of the manuscript. RR was in charge of the statistical analyses. All authors contributed to interpretation of data and critical revision of the manuscript. All authors gave final approval of the work to be published.

\section{Ethics approval and consent to participate}

The research committee of the of Helsinki University Hospital (HUS/26/2018 §37), the Finnish National Institute for Health and Welfare (THL/2034/5.05.00/ 2017), Statistics Finland (TK-53-1047-14), the Social Insurance Institution (Kela 23/522/2018), the Office of the Data Protection Ombudsman (2794/402/ 2015) and all of the participating university hospitals' ethics committees approved this study and waived the need for informed consent.

\section{Consent for publication}

Not applicable.

\section{Competing interests}

The authors declare that they have no competing interests.

\section{Publisher's Note}

Springer Nature remains neutral with regard to jurisdictional claims in published maps and institutional affiliations.

\section{Author details}

${ }^{1}$ Department of Neurosurgery, Helsinki University Hospital and University of Helsinki, Topeliuksenkatu 5, PB 266, 00029 HUS Helsinki, Finland.

${ }^{2}$ Department of Intensive Care, Kuopio University Hospital \& University of Eastern Finland, Kuopio, Finland. ${ }^{3}$ Department of Intensive Care, North Karelia Central Hospital, Joensuu, Finland. ${ }^{4}$ Department of Intensive Care, Tampere University Hospital \& University of Tampere, Tampere, Finland. ${ }^{5}$ Department of Intensive Care, Turku University Hospital \& University of Turku, Turku, Finland. ${ }^{6}$ Department of Intensive Care, Oulu University Hospital \& University of Oulu, Medical Research Center, Research Group of Surgery, Anesthesiology and Intensive Care, Oulu, Finland. ${ }^{7}$ Department of Neurology, Helsinki University Hospital \& University of Helsinki, Helsinki, Finland. ${ }^{8}$ Department Anesthesia, Intensive Care and Pain Medicine and Department of Emergency Care and Services, Helsinki University Hospital \& University of Helsinki, Helsinki, Finland.

Received: 15 April 2018 Accepted: 7 August 2018

Published online: 20 September 2018

\section{References}

1. Majdan M, Plancikova D, Maas A, Polinder S, Feigin V, Theadom A, et al. Years of life lost due to traumatic brain injury in Europe: a cross-sectional analysis of 16 countries. PLoS Med. 2017;14:e1002331. Schreiber M, editor. Public Library of Science. Available from: http://dx.plos.org/10.1371/journal. pmed.1002331. Cited 10 Sep 2017

2. Majdan M, Plancikova D, Brazinova A, Rusnak M, Nieboer D, Feigin V, et al. Epidemiology of traumatic brain injuries in Europe: a cross-sectional analysis. Lancet Public Health. 2016;1:e76-83. Available from: http:// linkinghub.elsevier.com/retrieve/pii/S2468266716300172. Cited 4 Mar 2017

3. GBD 2015 Neurological Disorders Collaborator Group VL, Abajobir AA, Abate $\mathrm{KH}, \mathrm{Abd}$-Allah F, Abdulle AM, Abera SF, et al. Global, regional, and national burden of neurological disorders during 1990-2015: a systematic analysis for the Global Burden of Disease Study 2015. Lancet Neurol. 2017;16:877-97. Elsevier. Available from: http://www.ncbi.n/m.nih.gov/pubmed/28931491. Cited 27 Jan 2018 
4. Feigin $\mathrm{VL}$, Roth GA, Naghavi M, Parmar P, Krishnamurthi R, Chugh S, et al. Global burden of stroke and risk factors in 188 countries, during 1990-2013: a systematic analysis for the Global Burden of Disease Study 2013. Lancet Neurol. 2016;15:913-24. Elsevier. Available from: http://www.ncbi.nlm.nih. gov/pubmed/27291521. Cited 27 Jan 2018

5. Halpern NA, Pastores SM. Critical care medicine in the United States 20002005: an analysis of bed numbers, occupancy rates, payer mix, and costs. Crit Care Med. 2010;38:65-71. Available from: https://www.ncbi.nlm.nih.gov/ pubmed/19730257. Cited 10 Mar 2017

6. Halpern NA, Pastores SM, Greenstein RJ. Critical care medicine in the United States 1985-2000: an analysis of bed numbers, use, and costs. Crit Care Med. 2004;32:1254-9. Available from: http://www.ncbi.nlm.nih.gov/pubmed/ 15187502. Cited 10 Mar 2017

7. Halpern NA, Goldman DA, Tan KS, Pastores SM. Trends in critical care beds and use among population groups and Medicare and Medicaid beneficiaries in the United States. Crit Care Med. 2016;44:1490-9. Available from: http://www.ncbi.nlm.nih.gov/pubmed/27136721. Cited 31 May 31

8. Reinikainen $M$, Uusaro A, Niskanen M, Ruokonen E. Intensive care of the elderly in Finland. Acta Anaesthesiol Scand. 2007;51:522-9.

9. Gyldmark M. A review of cost studies of intensive care units: problems with the cost concept. Crit Care Med. 1995;23:964-72. Available from: http:// www.ncbi.nlm.nih.gov/pubmed/7736758. Cited 31 May 2018

10. Reinikainen M, Mussalo P, Hovilehto S, Uusaro A, Varpula T, Kari A, et al. Association of automated data collection and data completeness with outcomes of intensive care. A new customised model for outcome prediction. Acta Anaesthesiol Scand. 2012;56:1114-22. Available from: http:// www.ncbi.nlm.nih.gov/pubmed/22384799. Cited 12 Sep 12

11. Keene AR, Cullen DJ. Therapeutic intervention scoring system: update 1983. Crit Care Med. 1983;11:1-3. Available from: http://www.ncbi.nlm.nih.gov/entrez/query. fcgi?cmd=Retrieve\&db=PubMed\&dopt=Citation\&list_uids=6848305

12. Knaus WA, Draper EA, Wagner DP, Zimmerman JE. APACHE II: A severity of disease classification system. Crit Care Med. 1985;13:818-29.

13. Le Gall J-R, Lemeshow S, Saulnier F. Simplified acute physiology score (SAPS II) based on a European/North American multicenter study. JAMA. 1993;270:2957-63.

14. Vincent JL, Moreno R, Takala J, Willatts S, De Mendonça A, Bruining H, et al. The SOFA (Sepsis-related Organ Failure Assessment) score to describe organ dysfunction/failure. On behalf of the Working Group on Sepsis-Related Problems of the European Society of Intensive Care Medicine. Intensive Care Med. 1996;22:707-10. Available from: http://www.ncbi.nlm.nih.gov/ pubmed/8844239. Cited 12 Sep 2017

15. Oken MM, Creech RH, Tormey DC, Horton J, Davis TE, McFadden ET, et al. Toxicity and response criteria of the Eastern Cooperative Oncology Group. Am J Clin Oncol. 1982;5:649-55. Available from: http://www.ncbi.nlm.nih. gov/pubmed/7165009. Cited 19 Feb 2017

16. Efendijev I, Raj R, Skrifvars MB, Hoppu S, Reinikainen M. Increased need for interventions predicts mortality in the critically ill. Acta Anaesthesiol Scand. 2016;60(10):1415-24. https://doi.org/10.1111/aas.12809.

17. Raj R, Bendel S, Reinikainen M, Hoppu S, Luoto T, Ala-Kokko T, et al. Temporal trends in healthcare costs and outcome following ICU admission after traumatic brain injury. Crit Care Med [internet]. 2018;1. Available from: http://www.ncbi.nlm.nih.gov/pubmed/29293155. Cited 26 Jan 2018

18. Kapiainen S, Väisänen A, Haula T, Raportti R. Terveyden-ja sosiaalihuollon yksikkökustannukset Suomessa vuonna 2011 [Internet]. 2014. Available from: http://www.julkari.fi/bitstream/handle/10024/114683/THL_RAPO3_2014_ web.pdf?sequence=1. Accessed 1 Jan 2017.

19. Raj R, Skrifvars M, Bendel S, Selander T, Kivisaari R, Siironen J, Reinikainen M. Predicting six-month mortality of patients with traumatic brain injury: usefulness of common intensive care severity scores. Crit Care. 2014;18(2): R60 URL: https://www.ncbi.nlm.nih.gov/pubmed/24708781.

20. Fallenius M, Skrifvars MB, Reinikainen M, Bendel S, Raj R. Common intensive care scoring systems do not outperform age and Glasgow coma scale score in predicting mid-term mortality in patients with spontaneous intracerebral hemorrhage treated in the intensive care unit. Scand J Trauma Resusc Emerg Med. 2017;25(1):102. https://doi.org/10.1186/s13049-017-0448-z.

21. Reynolds HN, Haupt MT, Thill-Baharozian MC, Carlson RW. Impact of critical care physician staffing on patients with septic shock in a university hospital medical intensive care unit. JAMA. 1988;260:3446-50. Available from: http:// www.ncbi.nlm.nih.gov/pubmed/3210284. Cited 10 Mar 2017

22. Fernando SM, Reardon PM, Dowlatshahi D, English SW, Thavorn K, Tanuseputro $P$, et al. Outcomes and costs of patients admitted to the ICU due to spontaneous intracranial hemorrhage. Crit Care Med [Internet]. 2018;1. Available from: https://www.ncbi.nlm.nih.gov/pubmed/29406421. Cited 11 Feb 2018

23. Wilby MJ, Sharp M, Whitfield PC, Hutchinson PJ, Menon DK, Kirkpatrick PJ. Cost-effective outcome for treating poor-grade subarachnoid hemorrhage. Stroke. 2003;34:2508-11. Available from: http://www.ncbi.nlm.nih.gov/ pubmed/12958321. Cited 5 Feb 2018

24. Maud A, Lakshminarayan K, MFK S, Vazquez G, Lanzino G, Qureshi Al. Costeffectiveness analysis of endovascular versus neurosurgical treatment for ruptured intracranial aneurysms in the United States. J Neurosurg. 2009;110: 880-6. American Association of Neurological Surgeons. Available from: http://thejns.org/doi/10.3171/2008.8.JNS0858. Cited 27 Mar 2018

25. Chen A, Bushmeneva K, Zagorski B, Colantonio A, Parsons D, Wodchis WP. Direct cost associated with acquired brain injury in Ontario. BMC Neurol. 2012;12:76. Available from: http://www.ncbi.n/m.nih.gov/pubmed/22901094. Cited 4 Mar 2017

26. Te Ao B, Brown P, Tobias M, Ameratunga S, Barker-Collo S, Theadom A, et al. Cost of traumatic brain injury in New Zealand: evidence from a population-based study. Neurology. 2014;83(18):1645-52. https://doi.org/10. 1212/WNL.0000000000000933.

27. Davis KL, Joshi AV, Tortella BJ, Candrilli SD. The direct economic burden of blunt and penetrating trauma in a managed care population. J Trauma. 2007;62:622-30. Available from: http://www.ncbi.nlm.nih.gov/pubmed/ 17414338. Cited 13 Mar 2017

28. Hemphill JC, Greenberg SM, Anderson CS, Becker K, Bendok BR, Cushman $M$, et al. Guidelines for the management of spontaneous intracerebral hemorrhage. Stroke [internet]. 2015; Available from: http://stroke.ahajournals. org/content/early/2015/05/28/STR.0000000000000069. Cited 12 Sept 2017

29. Jovin TG, Chamorro A, Cobo E, de Miquel MA, Molina CA, Rovira A, et al. Thrombectomy within 8 hours after symptom onset in ischemic stroke. $N$ Engl J Med. 2015;372:2296-306. Massachusetts Medical Society. Available from: http://www.nejm.org/doi/10.1056/NEJMoa1503780. Cited 13 Feb 2018

30. Saver $\mathrm{L}$, Goyal M, Bonafe A, Diener H-C, Levy El, Pereira VM, et al. Stentretriever thrombectomy after intravenous t-PA vs. t-PA alone in stroke. N Engl J Med. 2015;372:2285-95. Massachusetts Medical Society. Available from: http://www.nejm.org/doi/10.1056/NEJMoa1415061. Cited 13 Feb 2018

31. Campbell BCV, Mitchell PJ, Kleinig TJ, Dewey HM, Churilov L, Yassi N, et al. Endovascular therapy for ischemic stroke with perfusion-imaging selection. N Engl J Med. 2015;372:1009-18. Massachusetts Medical Society. Available from: http://www.nejm.org/doi/10.1056/NEJMoa1414792. Cited 13 Feb 2018

32. Goyal M, Demchuk AM, Menon BK, Eesa M, Rempel JL, Thornton J, et al. Randomized assessment of rapid endovascular treatment of ischemic stroke. N Engl J Med. 2015;372:1019-30. Massachusetts Medical Society. Available from: http://www.nejm.org/doi/10.1056/NEJMoa1414905. Cited 13 Feb 2018

33. Berkhemer OA, Fransen PSS, Beumer D, van den Berg LA, Lingsma HF, yoo a, et al. a randomized trial of intraarterial treatment for acute ischemic stroke. N Engl J Med. 2015;372:11-20. Available from: http://www.ncbi.nlm. nih.gov/pubmed/25517348. Cited 13 Feb 2018

34. Shireman TI, Wang K, Saver JL, Goyal M, Bonafé A, Diener H-C, et al. Costeffectiveness of Solitaire stent retriever thrombectomy for acute ischemic stroke. Stroke. 2017;48:379-87. Available from: http://www.ncbi.nlm.nih.gov/ pubmed/28028150. Cited 13 Feb 2018

35. Achit H, Soudant M, Hosseini K, Bannay A, Epstein J, Bracard S, et al. Costeffectiveness of thrombectomy in patients with acute ischemic stroke. Stroke. 2017;48:2843-7. Available from: http://www.ncbi.nlm.nih.gov/ pubmed/28916667. Cited 13 Feb 2018

36. Rhodes A, Ferdinande P, Flaatten H, Guidet B, Metnitz PG, Moreno RP. The variability of critical care bed numbers in Europe. Intensive Care Med. 2012; 38:1647-53. Springer-Verlag. Available from: https://www.ncbi.nlm.nih.gov/ pubmed/22777516. Cited 17 Mar 2018

37. Carr BG, Addyson DK, Kahn JM. Variation in critical care beds per capita in the United States: implications for pandemic and disaster planning. JAMA 2010;303:1371. Available from: http://www.ncbi.nlm.nih.gov/pubmed/ 20388892. Cited 17 Mar 2018

38. Korja M, Lehto $H$, Juvela $\mathrm{S}$, Kaprio J. Incidence of subarachnoid hemorrhage is decreasing together with decreasing smoking rates. Neurology. 2016;87: 1118-23. Available from: http://www.ncbi.nlm.nih.gov/pubmed/27521438. Cited 11 Feb 2017 